\section{Much ado about TDO}

\section{By Kai-Jye Lou, Staff Writer}

While companies are pursuing indolamine 2,3-dioxygenase inhibitors for cancer, German academics have identified an alternate tryptophan metabolism pathway mediated by an enzyme called tryptophan 2,3-dioxygenase that tumors exploit to grow and evade the immune system. ${ }^{1}$ The study suggests the need to monitor tumors for activation in both pathways.

Indolamine 2,3-dioxygenase (IDO)-mediated tryptophan catabolism generates kynurenine, a metabolite that inhibits the antitumor immune response. $^{2}$

At least two companies have inhibitors of IDO in Phase I testing for solid tumors. NewLink Genetics Corp. is developing 1-methyl-D-tryptophan, a selective inhibitor of IDO. Incyte Corp. is developing INCB24360.
Incyte previously ran high throughput screening and in vitro assays to identify compounds that inhibited IDO with little or no activity against tryptophan 2,3-dioxygenase (TDO2; TDO) to help decrease potential side effects. ${ }^{3-5}$ The company's desire to avoid TDO activity made sense, as the enzyme had not been previously linked to cancer and is expressed in healthy liver cells and neurons.

Meanwhile, Michael Platten began to suspect a role for TDO in cancer following his German team's studies on the immunoregulatory role of tryptophan metabolism in human diseases.

"Our past work in animal models has suggested that tryptophan metabolism suppresses the autoimmune response and could be significant for treating diseases like multiple sclerosis," said Platten, who is a professor in the Department of Neurooncology at Heidelberg University Hospital and head of the Experimental Neuroimmunology Unit at the German Cancer Research Center. "We also know that gliomas are particularly immunosuppressive tumors and are able to exert their immunosuppressive effects at both the local and systemic levels. Thus, we wanted to investigate the relevance of tryptophan metabolism to the immunosuppressive effects of glioma cells."

In human glioma cell lines and in glioma-initiating cells, constitutive tryptophan catabolism-and the resulting generation of

\section{Tumor cell}

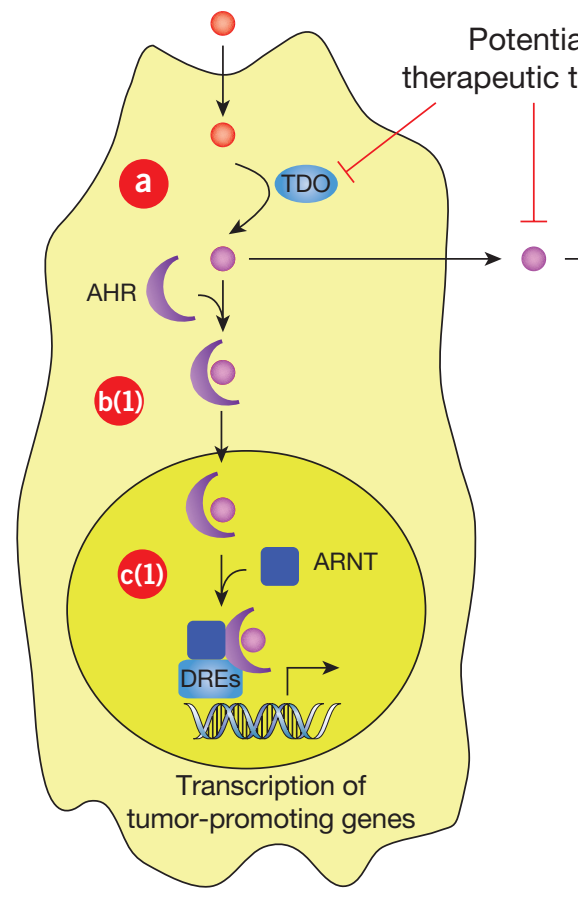

$\uparrow \begin{aligned} & \text { Growth } \\ & \text { Motility }\end{aligned}$
Immune cell

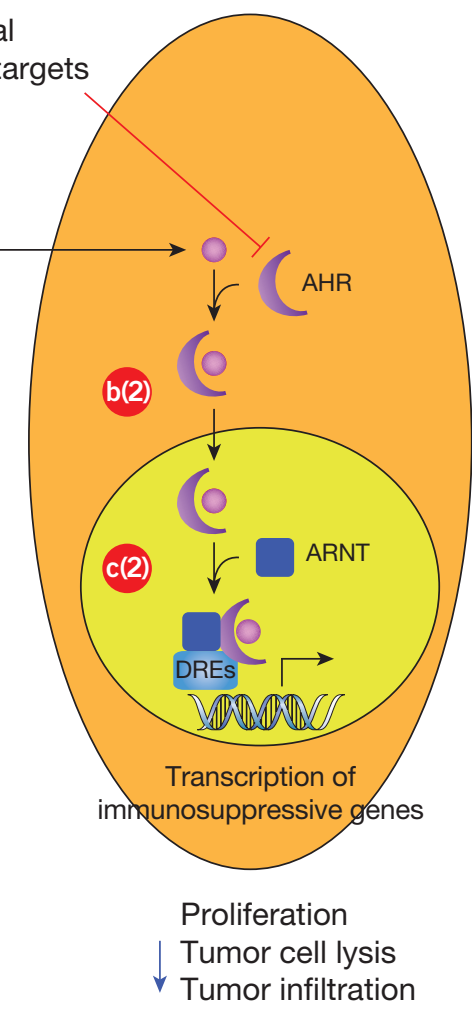

Figure 1. Model for TDO-derived kynurenine signaling through AHR. The tryptophan metabolite kynurenine is an endogenous ligand for the aryl hydrocarbon receptor (AHR) that can promote tumor cell growth, motility and immune escape.

Tryptophan (orange circle) enters the tumor cell and is degraded into kynurenine (purple circle) by tryptophan 2,3-dioxygenase (TDO2; TDO) [a(1)]. Kynurenine then binds to AHR (purple crescent), and the resulting ligand-receptor complex translocates into the nucleus $[\mathbf{b}(\mathbf{1})]$. Within the nucleus, the complex forms a heterodimer with AHR nuclear translocator (ARNT; HIF1b) (blue square), which then interacts with dioxin-responsive elements (DREs) to regulate the expression of AHR target genes that promote tumor cell growth and motility [c(1)].

Tumor cells also can suppress the antitumor immune response through the release of TDO-derived kynurenine. Similar to what happens in tumor cells, kynurenine binds to AHR in immune cells [b(2)], translocates into the cell nucleus and forms a heterodimer with ARNT that regulates AHR target genes that promote immunosuppression [c(2)].

The identified pathways implicate TDO, kynurenine and AHR as potential therapeutic targets. (Figure based on Figure $5 \mathrm{~g}$ in ref. 1.) 
kynurenine-was mediated by TDO and not by IDO.

In cell coculture and in mouse glioma models, TDO-derived kynurenine inhibited the antitumor immune response and promoted tumor cell growth and survival. A TDO deficiency blocked those effects compared with no deficiency.

Despite establishing a link between TDO and cancer, Platten thinks the enzyme itself may not be a great therapeutic target because of its presence in liver and brain cells. Thus, his team has looked downstream for more druggable targets.

In glioma cells, microarray analysis revealed that the 25 genes most strongly induced by kynurenine were regulated by the aryl hydrocarbon receptor (AHR). Radioligand binding assays and follow-up studies with an AHR antagonist and AHR knockdown confirmed that kynurenine binds to and activates AHR (see Figure 1, "Model for TDO-derived kynurenine signaling through AHR"). In mice injected with AHR-deficient human glioma cells, tumor growth was lower than that in mice given nondeficient glioma cells.

Importantly, additional microarray analyses suggested that the identified TDO-kynurenine-AHR signaling pathway is active in other types of cancer, including B cell lymphoma, Ewing's sarcoma and carcinomas in five different organs.

Results were published in Nature.

"Kynurenine signaling has previously been linked to the regulation of T cell activity, but its significance in cancer biology was not established," said George Prendergast, professor, president and CEO at the Lankenau Institute for Medical Research. "The reported findings are important, as they connect TDO-mediated kynurenine signaling to tumor growth and also show that it could be involved in immune escape."

"Our work shows that when looking at the culprit enzymes responsible for constitutive tryptophan metabolism in tumors, it's important to focus not only on IDO but on TDO as well," said Platten, who is corresponding author on the paper.

\section{Escape options}

Platten said the role of TDO-mediated tryptophan metabolism "is highly significant because there are inhibitors of IDO being tested in the clinic for cancer and they do not block signaling through TDO. We found that this TDO signaling pathway is active in gliomas and possibly several other tumor types, which suggests that there may be a need to inhibit tryptophan metabolism through this pathway in addition to through IDO.”
Prendergast, who has authored patents covering IDO inhibitors, thinks tumor immune escape is most likely to occur through either the IDO or TDO pathways rather than through both at the same time.

"Previous work has suggested that tumors can employ different strategies to escape the immune system," he told SciBX. "We know that some tumors use IDO for immune escape, and we see in the current study that others such as brain tumors may prefer to use TDO instead. It could be important going forward to determine which escape pathway is being exploited by a particular tumor to help determine which one to target to prevent immune escape."

Prendergast also suggested that AHR could be at a nodal point downstream of cancer signaling pathways mediated by IDO and TDO. Thus, targeting the receptor could potentially affect the cancerpromoting effects of both pathways.

"It wouldn't be surprising if others found that IDO also facilitates tumor immune escape through AHR," he told SciBX.

Regardless, Prendergast said additional genetic validation studies will be needed to determine whether TDO and AHR would be good therapeutic targets.

NewLink and Incyte both declined to comment.

Platten's group is screening for compounds that target and inhibit kynurenine signaling through AHR. He declined to disclose the patent and licensing information associated with the group's work.

Lou, K.-J. SciBX 4(42); doi:10.1038/scibx.2011.1165

Published online Oct. 27, 2011

\section{REFERENCES}

1. Opitz, C.A. et al. Nature; published online Oct. 6, 2011; doi:10.1038/nature10491

Contact: Michael Platten, Heidelberg University Hospital, Heidelberg, Germany e-mail: m.platten@dkfz-heidelberg.de

2. Prendergast, G.C. Oncogene 27, 3889-3900 (2008)

3. Yue, E.W. et al. J. Med. Chem. 52, 7364-7367 (2009)

4. Haas, M.J. SciBX 2(26); doi:10.1038/scibx.2009.1029

5. Koblish, H.K. et al. Mol. Cancer Ther. 9, 489-498 (2010)

\section{COMPANIES AND INSTITUTIONS MENTIONED}

German Cancer Research Center, Heidelberg, Germany Heidelberg University Hospital, Heidelberg, Germany Incyte Corp. (NASDAQ:INCY), Wilmington, Del. Lankenau Institute for Medical Research, Wynnewood, Pa. NewLink Genetics Corp., Ames, lowa 